\title{
Plasma desacyl ghrelin-to-acyl ghrelin ratio is a predictor of postoperative complications and prognosis after pancreaticoduodenectomy
}

\author{
TAKAHIRO NISHIDA ${ }^{1}$, HIRONOBU TSUBOUCHI ${ }^{2}$, TAKEOMI HAMADA ${ }^{3}$, \\ NAOYA IMAMURA ${ }^{3}$, MASAHIDE HIYOSHI ${ }^{3}$, KOICHI YANO $^{3}$, KENJI KANGAWA ${ }^{4}$, \\ MASAMITSU NAKAZATO ${ }^{2}$ and ATSUSHI NANASHIMA ${ }^{3}$
}

\begin{abstract}
${ }^{1}$ Division of Gastrointestinal, Endocrine and Pediatric Surgery, Department of Surgery; ${ }^{2}$ Division of Neurology, Respirology, Endocrinology and Metabolism, Department of Internal Medicine; ${ }^{3}$ Division of Hepato-Biliary-Pancreatic Surgery,

Department of Surgery, University of Miyazaki Faculty of Medicine, Miyazaki 889-1692;

${ }^{4}$ Department of Biochemistry, National Cardiovascular Center Research Institute, Osaka 565-8565, Japan
\end{abstract}

Received March 29, 2019; Accepted July 1, 2019

DOI: $10.3892 / 01.2019 .10821$

\begin{abstract}
The current study aimed to clarify the significance of the preoperative desacyl ghrelin (DG)-to-acyl ghrelin (AG) ratio in patients undergoing pancreaticoduodenectomy $(\mathrm{PD})$. Ghrelin, a peptide hormone mainly produced in the stomach, possesses unique functions. Recently, studies have determined the involvement of plasma gherlin in certain postoperative outcomes, particularly in surgical resections of the stomach. Although PD involves gastric resection, few reports have determined the involvement of ghrelin following PD. From April 2003 to December 2011, 195 patients underwent PD for tumors of the pancreatic head, bile duct and ampulla of Vater at the Division of Hepato-Biliary-Pancreatic Surgery, Department of Surgery, University of Miyazaki Faculty of Medicine (Miyazaki, Japan). Of these, 83 patients were enrolled into the present study as their plasma gherlin levels were measured.
\end{abstract}

Correspondence to: Professor Atsushi Nanashima, Division of Hepato-Biliary-Pancreatic Surgery, Department of Surgery, University of Miyazaki Faculty of Medicine, 5200 Kihara Road, Kiyotake, Miyazaki 889-1692, Japan

E-mail: a_nanashima@med.miyazaki-u.ac.jp

Abbreviations: AG, acyl ghrelin; $\mathrm{BMI}$, body mass index; $\mathrm{CD}$, Clavien-Dindo score; CEA, carcinoembryonic antigen; CRP, C-reactive protein; D/A, DG-to-AG ratio; DG, desacyl ghrelin; GEM, gemcitabine; GHSR, growth hormone-secretagogue receptor; HS-mGPS, high-sensitivity modified Glasgow Prognostic Score; OS, overall survival; PD, pancreaticoduodenectomy; PNI, prognostic nutritional index; PPPD, pylorus-preserving pancreaticoduodenectomy; ROC, receiver operating characteristic; SPD, standard pancreaticoduodenectomy; SSPPD, subtotal stomach-preserving pancreaticoduodenectomy; TG, total ghrelin

Key words: pancreaticoduodenectomy, acyl ghrelin, desacyl ghrelin, desacyl ghrelin-to-acyl ghrelin ratio, prognosis
AG, DG, total ghrelin (TG) and the DG-to-AG ratio (D/A) were subsequently assessed. Furthermore, the prognostic nutritional index (PNI) and high-sensitivity modified Glasgow Prognostic Score (HS-mGPS) were determined. Morbidity was examined in all 83 patients, but mortality was only determined in 69 individuals after 14 patients were excluded due to the presence of benign disease. The results revealed that the TG of patients undergoing standard PD (SPD) was significantly lower than that of patients undergoing pylorus-preserving PD or subtotal stomach-preserving PD. It was also determined that TG levels declined significantly in SPD patients at 2 weeks after surgery. Negative associations were identified between plasma ghrelin levels and PNI, and between serum albumin and HS-mGPS. Patient morbidity was determined to be $31.3 \%$ and the severe complications exhibited by patients included pancreatic fistula (14.5\%), intra-abdominal abscess (15.7\%), intra-abdominal bleeding (6.0\%) and liver abscess (1.2\%). Multivariate analysis revealed that disease location and low D/A were independent risk factors for severe complications. The 5-year overall survival (OS) rate was $41.5 \%$. Multivariate analysis also demonstrated that diabetes mellitus, long postoperative hospital stay and low D/A were independent risk factors for OS. The present study revealed the D/A may serve as a useful predictive factor for postoperative complications and prognosis after PD.

\section{Introduction}

Pancreaticoduodenectomy (PD) is performed for tumors of the pancreatic head, bile duct and ampulla of Vater. PD has a high risk of postoperative pancreatic fistula, intra-abdominal abscess and bleeding due to the fistula and delayed gastric emptying $(1,2)$, which causes malnutrition and impairs quality of life. Complications related to postoperative pancreatic fistula can be fatal. Ever since the reports by Watson in 1944 (3) and Traverso and Longmire in 1978 (4), reduction surgeries such as pylorus-preserving PD (PPPD), subtotal stomach-preserving PD (SSPPD) and duodenum-preserving pancreatic head resection have been performed actively for organ preservation. The 
standard PD (SPD) tends to be selected only for patients with simultaneous gastric cancer or history of gastrectomy and patients with tumors of the pancreatic head region involving the stomach. To reduce complications after PD, the usefulness of these reduction surgeries and perioperative predictive factors have been investigated $(1,2,5,6)$.

In recent years, the involvement of plasma ghrelin in postoperative outcomes has been reported. Ghrelin was discovered as an intrinsic ligand for the growth hormone-secretagogue receptor (GHSR) in 1999 by Kojima et al (7). Ghrelin is peptide hormone mainly produced in the stomach and has an active form acylated by ghrelin O-acyltransferase and a desacyl form, which is a degradation product. In healthy humans, the blood concentration of desacyl ghrelin (DG) is about 4-5 times that of acyl ghrelin (AG). AG has unique functions such as growth hormone secretion, promotion of food intake and gastrointestinal motility, gastric acid secretion, weight gain action and strong anti-inflammatory action (7-12). In contrast, DG is not involved in growth hormone secretion activity, but there are reports of actions on the promotion or suppression of food intake, an anti-arteriosclerotic effect, suppression of inflammatory cell infiltration, protection against muscle atrophy, neuroprotection and others $(13,14)$.

Depending on the procedure, PD can involve resection of the stomach. However, studies on plasma ghrelin in patients undergoing PD are rare. This study aimed to clarify the significance of the perioperative plasma ghrelin level in PD patients.

\section{Patients and methods}

Patients. This study is retrospective study. It was approved by the Human Ethics Review Board of the University of Miyazaki on 2018/11/21 (reference number O-0426), and it conforms to the provisions of the Declaration of Helsinki. Informed consent for data collection was obtained from the patients using the opt-out procedure. Patient data were retrieved from the departmental database. Between April 2003 and December 2011, 195 patients underwent PD for hepatobiliary pancreatic disease at the Division of Hepato-Biliary-Pancreatic Surgery, Department of Surgery, University of Miyazaki Faculty of Medicine in Japan. Plasma ghrelin was measured in 83 of these patients. Among them, 14 patients had benign disease (tumors and chronic inflammatory disease), and 69 had malignant tumors. Short-term outcomes were evaluated in all 83 patients, and long-term outcomes were evaluated in 69 patients after excluding those with benign disease. To assess patient condition, we used the following indices to respectively score combined immunonutrition and inflammation status: The prognostic nutritional index (PNI) and the high-sensitivity modified Glasgow Prognostic Score (HS-mGPS) (15-18). The following factors were examined: Age, sex, body mass index (BMI), diabetes mellitus, preoperative biliary drainage, nutritional score (PNI), inflammation-based prognostic score (HS-mGPS), serum tumor markers [carcinoembryonic antigen (CEA), carbohydrate antigen 19-9], tumor location, tumor type, tumor size, pathological status based on the international Union Against Cancer (UICC) tumor-node-metastasis (TNM) classification (8th edition) (19), surgical procedure (SPD, PPPD, SSPPD), portal vein resection, operation time, blood loss, severe complications, postoperative hospital stay, adjuvant chemotherapy, plasma ghrelin level and DG-to-AG ratio (D/A). We used the median for the basic values for the analysis and the cut-off values derived from receiver operating characteristic (ROC) analysis for the tumor markers, plasma ghrelin and D/A. The PNI was calculated as $10 \mathrm{x}$ albumin $(\mathrm{g} / \mathrm{dl})+0.005 \mathrm{x}$ lymphocyte count $(/ \mu \mathrm{l})$. The HS-mGPS was determined based on C-reactive protein (CRP) and albumin levels. The scoring system was as follows: (1) Patients with a normal CRP value $(<0.3 \mathrm{mg} / \mathrm{dl})$ were allocated a score of 0 , regardless of the albumin level; (2) patients with a CRP level $\geq 0.3 \mathrm{mg} / \mathrm{dl}$ combined with an albumin level $\geq 3.5 \mathrm{~g} / \mathrm{dl}$ were allocated a score of 1 ; and (3) patients with a CRP $\geq 0.3 \mathrm{mg} / \mathrm{dl}$ and an albumin $<3.5 \mathrm{~g} / \mathrm{dl}$ were allocated a score of 2 . Postoperative complications were classified using the Clavien-Dindo grading system (20), where grade III or higher represents morbidity.

Measurement of plasma ghrelin levels. Blood samples were collected in a tube containing aprotinin and ethylenediaminetetraacetic acid (Wako, Osaka, Japan) before breakfast after an overnight fast. Plasma total ghrelin (TG) concentrations were calculated as the AG concentration plus the DG concentration. Blood sampling points were preoperatively, 2 weeks, and 1, 6 and 12 months after surgery.

Surgical procedure. The same surgical team performed all operations and oversaw the perioperative management. The operative technique was essentially similar to that described previously (21). The organs resected during PD included the gallbladder, common hepatic duct, head of the pancreas, duodenum except for a $3-4 \mathrm{~cm}$ portion nearby the bulb and approximately $10 \mathrm{~cm}$ of the proximal jejunum. The pancreas was divided with a scalpel. The duodenum was transected approximately 3-4 cm distal to the pyloric ring for PPPD, and the stomach was transected approximately $2-3 \mathrm{~cm}$ proximal to the pyloric ring at the antrum in SSPPD. We performed pancreatojejunostomy by the duct-to-mucosa method with eight interrupted sutures of 5-0 PDS-II (polydioxanone; Johnson \& Johnson Co.) and the modified Kakita method with 3-0 Prolene (polypropylene; Johnson \& Johnson Co.) (22). An end-to-side hepaticojejunostomy was then performed $5-10 \mathrm{~cm}$ distal from the pancreatojejunostomy. Finally, end-to-side duodeno- or gastrojejunostomy was performed to place the stomach and duodenum into a straight vertical line as described for the retrocolic route or the antecolic route, which was decided by randomized controlled trial (23). A Braun anastomosis was then created. Closed drains were inserted behind the hepaticojejunostomy and at the upper side of the pancreatojejunostomy. All patients received prophylactic antibiotics for 3 days and an $\mathrm{H}_{2}$ blocker or proton pump inhibitor to prevent stress peptic ulcer. None of the patients received octreotide.

Adjuvant chemotherapy. In 2016, the Japan Adjuvant Study Group of Pancreatic Cancer (JASPAC) reported the results of a phase III study of S-1 vs. gemcitabine (GEM) for resected pancreatic cancer. S-1 significantly prolonged overall survival (OS) and relapse-free survival, and S-1 therapy is currently recommended (24). At the time of the present study, there was no evidence on adjuvant therapy for pancreatic cancer, and various methods such as S-1, GEM or S-1+GEM were selected. 
Table I. Clinicopathological characteristics of patients undergoing SPD and non-SPD.

\begin{tabular}{lccr}
\hline Variables & SPD $(\mathrm{n}=9)$ & Non-SPD $(\mathrm{n}=74)$ & P-value \\
\hline Age, years [median (range)] & $76(61-79)$ & $69(36-85)$ & 0.1220 \\
Sex (male/female) & $7 / 2$ & $42 / 32$ & 0.2260 \\
BMI, kg/m² [median, (range)] & $19.5(16.3-24.4)$ & $20.8(13.8-29.0)$ & 0.3448 \\
Diabetes mellitus & $3(33.3 \%)$ & $32(43.2 \%)$ & 0.5697 \\
Preoperative biliary drainage & $3(33.3 \%)$ & $47(63.5 \%)$ & 0.0807 \\
PNI [median, (range)] & $44.1(42.3-49.2)$ & $43.7(29.4-51.4)$ & 0.6102 \\
HS-mGPS (0/1/2) & $7 / 2 / 0$ & $48 / 10 / 16$ & 0.2768 \\
Tumor location (pancreas/bile duct/ampulla) & $5 / 3 / 1$ & $42 / 21 / 11$ & 0.9285 \\
Tumor type (malignant/benign) & $9 / 0$ & $60 / 14$ & 0.1524 \\
Portal vein resection n(\%) & $3(33.3 \%)$ & $11(14.9 \%)$ & 0.1624 \\
Operation time, min [median, (range)] & $675(552-726)$ & $579(427-967)$ & 0.0514 \\
Blood loss, ml [median (range)] & $1800(1150-3040)$ & $1455(440-6870)$ & 0.2472 \\
CD score $\geq I I I$ n(\%) & $5(55.6 \%)$ & $21(28.4 \%)$ & 0.0970 \\
Postoperative hospital stay, days [median (range)] & $37(29-49)$ & $36(24-116)$ & 0.9649 \\
Preoperative ghrelin, fmol/ml [median (range)] & & & \\
AG & $2.19(1.21-12.41)$ & $7.28(0.15-59.36)$ & 0.0250 \\
DG & $20.31(4.49-66.60)$ & $44.26(3.08-306.39)$ & 0.0994 \\
Total ghrelin & $22.47(6.70-79.01)$ & $54.70(4.36-313.65)$ & 0.0489 \\
DG-to-AG ratio & $6.00(2.03-34.34)$ & $4.36(1.05-142.11)$ & 0.3087 \\
\hline
\end{tabular}

SPD, standard pancreaticoduodenectomy; BMI, body mass index; PNI, prognostic nutritional index; HS-mGPS, high-sensitivity modified Glasgow Prognostic Score; CD, Clavien-Dindo score; DG, desacyl ghrelin; AG, acyl ghrelin.

Evidence for adjuvant chemotherapy for biliary carcinoma and ampullary carcinoma was still lacking at the time GEM or GEM+cisplatin were chosen.

Statistical analysis. Clinical parameters are expressed as the number $(\%)$ or median value and range. Comparisons between groups were made using the $\chi^{2}$ test for categorical variables and the Mann-Whitney U test for continuous variables. The difference between plasma ghrelin concentrations at different time-points was tested for significance using a Friedman test followed by a post hoc Bonferroni correction. Survival was analyzed using the Kaplan-Meier method, and the log-rank test was used to determine the differences between the two groups. The cutoff for definition of a subgroup was determined by a ROC analysis. A univariate analysis was performed to assess significant differences in clinicopathological characteristics. The independent risk factors affecting severe complications and prognostic factors were determined by multivariate analysis using a Cox proportional hazards model for variables that were significant following univariate analysis. The associated $95 \%$ confidence interval (CI) was subsequently determined. $\mathrm{P}<0.05$ was considered statistically significant. Statistical analyses were performed using JMP $11^{\circledR}$ (SAS Institute Inc., Cary, NC, USA).

\section{Results}

Clinicopathological characteristics. PD was performed in 83 patients (49 men, 34 women) with a median age of 70 (range 36-85) years: 63 underwent PPPD, 11 SSPPD and 9 SPD for diagnoses that included pancreatic disease $(n=47)$, bile duct disease $(n=24)$ and ampullary tumor $(n=12)$. The clinicopathological characteristics of the SPD group $(n=9)$ and Non-SPD group $(n=74)$ are summarized in Table I. Of the 83 patients, 69 had a malignant tumor (pancreatic carcinoma: 36 , bile duct carcinoma: 22, ampullary carcinoma: 11 patients). Thirty-three patients $(47.8 \%)$ had diabetes mellitus, and $44(63.8 \%)$ required biliary drainage for preoperative jaundice and cholangitis. The progression of cancer by TNM classification tended toward advanced cases (Stage I: 9, II: 25, III: 35 patients). The clinicopathological characteristics of the pancreatic carcinoma group (PCG, $n=36$ ) and the non-pancreatic carcinoma group (Non-PCG, $n=33$ ) are summarized in Table II. The PCG included many patients with deeper invasive lesions $(\mathrm{P}=0.0156)$ and portal vein resection $(38.9 \%$ vs. $0.0 \%, \mathrm{P}<0.0001)$ and adjuvant chemotherapy $(86.1 \%$ vs. $63.6 \%, \mathrm{P}=0.0304)$. In the Non-PCG, more patients required preoperative biliary drainage than in the PCG (78.8\% vs. $50.0 \%, \mathrm{P}=0.0130)$.

Plasma ghrelin and immunonutritional status. Fig. 1 shows the chronological change of plasma TG and D/A after PD. The TG in patients who underwent SPD was significantly lower than that in those who underwent PPPD or SSPPD (Fig. 1A). In SPD patients, TG had declined significantly at 2 weeks after surgery (mean, preoperative 32.8 vs. 2 weeks postoperatively $15.9 \mathrm{fmol} / \mathrm{ml}, \mathrm{P}=0.0388$ ). There were no significant differences at other times points. Table I shows the significant difference between the SPD group and the Non-SPD group in AG and TG, but shows no difference in the D/A. The perioperative D/A declined significantly at 
Table II. Clinicopathological characteristics of the PCG and non-PCG patients.

\begin{tabular}{|c|c|c|c|}
\hline Variables & PCG $(n=36)$ & Non-PCG $(\mathrm{n}=33)$ & P-value \\
\hline Age, years [median (range)] & $68.5(43-85)$ & $69.0(51-82)$ & 0.9091 \\
\hline Sex (male/female) & $15 / 21$ & $24 / 9$ & 0.0093 \\
\hline BMI, $\mathrm{kg} / \mathrm{m}^{2}$ [median (range)] & $20.7(13.8-28.4)$ & $22.3(16.3-29.0)$ & 0.4208 \\
\hline Diabetes mellitus $\mathrm{n}(\%)$ & $21(58.3 \%)$ & $12(36.4 \%)$ & 0.0680 \\
\hline Preoperative biliary drainage $\mathrm{n}(\%)$ & $18(50.0 \%)$ & $26(78.8 \%)$ & 0.0130 \\
\hline PNI [median (range)] & $43.7(29.4-51.4)$ & $43.6(33.9-50.6)$ & 0.9605 \\
\hline HS-mGPS (0/1/2) & $26 / 4 / 6$ & $19 / 6 / 8$ & 0.4388 \\
\hline \multicolumn{4}{|l|}{ Tumor marker } \\
\hline CEA, ng/ml [median (range)] & $2.8(0.6-17.6)$ & $2.4(0.8-10.5)$ & 0.4276 \\
\hline CA19-9, U/ml [median, (range)] & $100.4(1.0-8132.8)$ & $32.0(2.0-31462)$ & 0.1598 \\
\hline Tumor size, mm [median, (range)] & $30(3-85)$ & $26(15-63)$ & 0.2604 \\
\hline Pathological depth of invasion (T1/T2/T3/T4) & $5 / 1 / 13 / 17$ & $4 / 10 / 10 / 9$ & 0.0156 \\
\hline Pathological nodal stage (N0/N1/N2) & $15 / 12 / 9$ & $17 / 12 / 4$ & 0.3826 \\
\hline Metastatic lymph nodes, n [median, (range)] & $1(0-25)$ & $0(0-6)$ & 0.1208 \\
\hline Stage (I/II/III) & $4 / 10 / 22$ & $5 / 15 / 13$ & 0.0904 \\
\hline Performed operation (SPD/PPPD/SSPPD) & $5 / 22 / 9$ & $4 / 27 / 2$ & 0.0840 \\
\hline Portal vein resection $\mathrm{n}(\%)$ & $14(38.9 \%)$ & $0(0 \%)$ & $<0.0001$ \\
\hline Operation time, min [median, (range)] & $654(450-967)$ & $580(432-934)$ & 0.6096 \\
\hline Blood loss, ml [median, (range)] & $1520(460-6870)$ & $1800(700-5930)$ & 0.3158 \\
\hline $\mathrm{CD}$ score $\geq \mathrm{III} \mathrm{n}(\%)$ & $2(5.6 \%)$ & $18(54.6 \%)$ & $<0.0001$ \\
\hline Postoperative hospital stay, days [median, (range)] & $33(25-49)$ & $43(25-116)$ & 0.0012 \\
\hline Adjuvant chemotherapy n(\%) & $31(86.1 \%)$ & $21(63.6 \%)$ & 0.0304 \\
\hline \multicolumn{4}{|l|}{ Preoperative ghrelin, fmol/ml, [median, (range)] } \\
\hline $\mathrm{AG}$ & $7.09(0.86-21.78)$ & $6.30(0.55-59.36)$ & 0.8288 \\
\hline DG & $45.88(3.27-120.85)$ & $40.75(4.49-306.39)$ & 0.7502 \\
\hline Total ghrelin & $54.70(4.89-128.15)$ & $46.57(6.70-313.65)$ & 0.8806 \\
\hline DG-to-AG ratio & $4.94(1.19-40.90)$ & $4.92(1.05-142.11)$ & 0.9665 \\
\hline
\end{tabular}

PCG, pancreatic carcinoma group; BMI, body mass index; PNI, prognostic nutritional index; HS-mGPS, high-sensitivity modified Glasgow Prognostic Score; CEA, carcinoembryonic antigen; CA19-9, carbohydrate antigen 19-9; SPD, standard pancreaticoduodenectomy; PPPD, pylorus-preserving pancreaticoduodenectomy; SSPPD, subtotal stomach-preserving pancreaticoduodenectomy; CD, Clavien-Dindo score; AG, acyl ghrelin; DG, desacyl ghrelin.

6 months after surgery in patients with SPD (mean; preoperative, 10.6 vs. 6 months postoperatively, $4.5 ; \mathrm{P}=0.0234$ ), but there were no significant differences at other time points (Fig. 1B). In all 83 patients, the median preoperative values of AG, DG, TG and D/A were 6.49, 40.86, $47.33 \mathrm{fmol} / \mathrm{ml}$ and 4.92. The cutoff values for morbidity of AG, DG, TG and D/A calculated by ROC analysis were 10.6, 41.6, $47.3 \mathrm{fmol} / \mathrm{ml}$ and 3.1. The median PNI was 43.7 , and 55 patients $(66.3 \%)$ had a HS-mGPS score of 0 . The plasma ghrelin levels of the high PNI group (PNI $\geq 40$ ) were significantly lower than those of the low PNI group (PNI <40) and showed a negative association. The D/A of the high PNI group (PNI $\geq 40$ ) tended to be higher than low PNI group, but there was no significant difference (Fig. 2A). The TG level in the patients with a HS-mGPS score of 1 was significantly lower than that of those with a score of 2 (score 1: 26.9 vs. score 2: $78.8 \mathrm{fmol} / \mathrm{ml}, \mathrm{P}=0.0451)$. The $\mathrm{D} / \mathrm{A}$ in the patients with a HS-mGPS score of 0 tended to be higher than other score group, but there was no significant difference (Fig. 2B).
Surgical outcomes. In the 83 patients, the median operation time and blood loss were $607 \mathrm{~min}$ and $1520 \mathrm{ml}$, respectively. The morbidity rate was $31.3 \%$, and the severe complications were pancreatic fistula $(14.5 \%)$, intra-abdominal abscess $(15.7 \%)$, intra-abdominal bleeding $(6.0 \%)$ and liver abscess (1.2\%). As shown in Table I, the Non-PCG had more severe complications than the PCG $(54.6 \%$ vs. $5.6 \%, \mathrm{P}<0.0001)$.

Univariate and multivariate analyses of clinical factors associated with morbidity. Clinical factors affecting severe complications were evaluated by univariate and multivariate analyses (Table III). A univariate analysis showed that sex [male, hazard ratio (HR): 3.22, $\mathrm{P}=0.0291$ ], location of the disease (not pancreas, HR: $8.54, \mathrm{P}<0.0001$ ) and $\mathrm{D} / \mathrm{A}$ (<3.1, HR: $6.23, \mathrm{P}=0.0018)$ were significant variables influencing unfavorable morbidity. Among them, location of the disease (not pancreas, HR: 9.64, $\mathrm{P}=0.0005)$ and $\mathrm{D} / \mathrm{A}(<3.1$, HR: 5.34, $\mathrm{P}=0.0292$ ) remained as independent risk factors for severe complications in a subsequent multivariate analysis. 
A
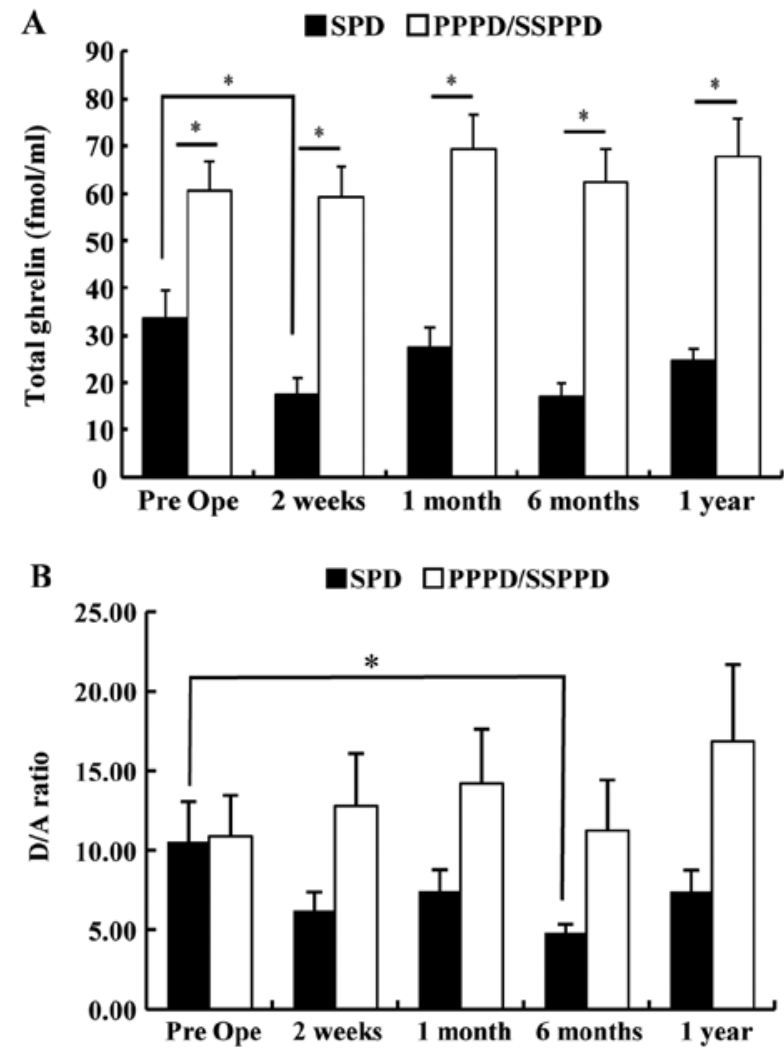

Figure 1. Chronological change of total plasma ghrelin and D/A after pancreatoduodenectomy. (A) The perioperative ghrelin levels of patients who underwent SPD were significantly lower than that those who underwent PPPD or SSPPD. In patients with SPD, total ghrelin levels decreased significantly at 2 weeks after surgery. (B) Perioperative D/A decreased significantly at 6 months after surgery in SPD patients. ${ }^{*} \mathrm{P}<0.05$ as indicated. D/A, desacyl ghrelin-to-acyl ghrelin ratio; SPD, standard pancreatoduodenectomy; PPPD, pylorus-preserving pancreatoduodenectomy; SSPPD, subtotal stomach-preserving pancreatoduodenectomy; Pre Ope, preoperative.

Survival analyses. Long-term results were examined in the 69 patients with malignant tumor. The rate of 5 -year OS in all patients was $41.5 \%$. The OS rates of the PCG and Non-PCG were 40.7 and $42.4 \%$, respectively, and the survival rate between the two groups was not significantly different $(\mathrm{P}=0.8773$; Fig. 3A). The survival rate between the SPD group and PPPD/SSPPD group was also not significantly different ( $44.4 \%$ vs. $41.0 \%, \mathrm{P}=0.8199$; Fig. $3 \mathrm{~B}$ ).

Univariate and multivariate analyses of prognostic factors. Factors associated with OS were evaluated by univariate and multivariate analyses (Table IV). A univariate analysis showed that diabetes mellitus (Yes, $\mathrm{P}=0.0001)$, CEA $(\geq 4.6 \mathrm{ng} / \mathrm{ml}$, $\mathrm{P}=0.0301)$, pathological depth of invasion $(\mathrm{T} 4, \mathrm{P}=0.0043)$, performed operation (PPPD, $\mathrm{P}=0.0313)$, blood loss ( $\geq 1565 \mathrm{~g}$, $\mathrm{P}=0.0103)$, postoperative hospital stay ( $\geq 36$ days, $\mathrm{P}=0.0144$ ) and $\mathrm{D} / \mathrm{A}(<3.1, \mathrm{P}=0.0012)$ were significant variables influencing unfavorable OS. Among them, diabetes mellitus (Yes, HR: 3.47, $\mathrm{P}=0.0014$ ), postoperative hospital stay ( $\geq 36$ days, HR: 2.28, $\mathrm{P}=0.0371)$ and $\mathrm{D} / \mathrm{A}(<3.1, \mathrm{HR}: 4.73, \mathrm{P}=0.0010)$ remained as independent risk factors for OS in a subsequent multivariate analysis. The rates of OS of the low D/A group (D/A <3.1) and high $\mathrm{D} / \mathrm{A}$ group $(\mathrm{D} / \mathrm{A} \geq 3.1)$ were 15.4 and $47.6 \%$, respectively $(\mathrm{P}=0.0134)$. In the patients of the PCG and Non-PCG, the rates
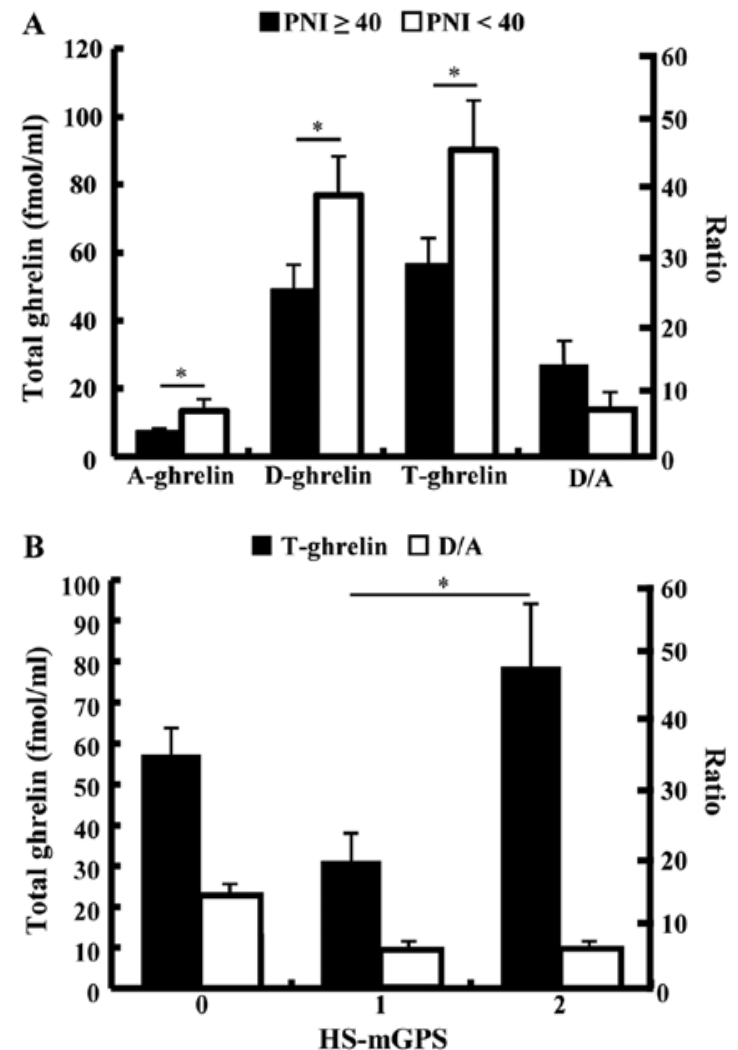

Figure 2. Association between PNI and plasma ghrelin levels or D/A, and between HS-mGPS) and total plasma ghrelin or D/A. (A) PNI and plasma ghrelin levels demonstrated a negative association. (B) Total ghrelin levels in patients with an HS-mGPS score of 1 were significantly lower than those patients with a score of $2 .{ }^{*} \mathrm{P}<0.05$ as indicated. D/A, desacyl ghrelin-to-acyl ghrelin ratio; PNI, prognostic nutritional index; D/A, desacyl ghrelin-to-acyl ghrelin ratio; HS-mGPS, high-sensitivity modified Glasgow Prognostic Score; A, acyl; D, desacyl; T, total.

of OS between the low D/A group and high D/A group were $0.0 \%$ vs. $50.8 \%(\mathrm{P}=0.0040)$ and $11.1 \%$ vs. $50.0 \%(\mathrm{P}=0.0247)$, respectively, and were significantly different (Fig. 4A and B).

\section{Discussion}

There are many reports on plasma ghrelin after gastrectomy and esophagectomy $(10,12)$, but there are very few reports after PD. Until now, perioperative change of the plasma ghrelin level, such as transient suppression during the day following pancreatectomy and its significance as a predictive marker of postoperative complications have been reported $(25,26)$. The present study is the first report, to our knowledge, to evaluate plasma ghrelin in relation to long-term outcomes.

In the present study, the plasma ghrelin levels did not change significantly over a one-year period, but the ghrelin level in the SPD patients was significantly lower than that in the PPPD/SSPPD patients. Seven of the SPD patients had undergone distal gastrectomy for gastric cancer in the past, and their ghrelin levels were already low prior to the SPD. Postoperatively, plasma ghrelin tends to recover to a normal level with time in patients who have undergone distal gastrectomy (27). Nine patients in this study underwent PD a median 6 (range 2-47) years after gastrectomy. Although Helicobacter pylori infection was not investigated in this 
Table III. Univariate and multivariate analyses of clinical factors affecting severe complications $(\mathrm{n}=83)$.

\begin{tabular}{|c|c|c|c|c|c|c|}
\hline \multirow[b]{2}{*}{ Variables } & \multicolumn{3}{|c|}{ Univariate } & \multicolumn{3}{|c|}{ Multivariate } \\
\hline & $\mathrm{HR}$ & $95 \% \mathrm{CI}$ & P-value & HR & $95 \% \mathrm{CI}$ & P-value \\
\hline Age $(\geq 70$ years $)$ & 2.42 & $0.92-6.33$ & 0.0722 & & & \\
\hline Sex (male) & 3.22 & $1.13-9.19$ & 0.0291 & 1.99 & $0.56-7.06$ & 0.2857 \\
\hline $\operatorname{BMI}\left(<20.8 \mathrm{~kg} / \mathrm{m}^{2}\right)$ & 1.04 & $0.41-2.62$ & 0.9409 & & & \\
\hline Diabetes mellitus (yes) & 2.62 & $0.95-7.20$ & 0.0616 & & & \\
\hline Preoperative biliary drainage (yes) & 1.76 & $0.66-4.70$ & 0.2610 & & & \\
\hline $\mathrm{PNI}(<40.0)$ & 0.75 & $0.18-3.05$ & 0.6876 & & & \\
\hline HS-mGPS (1+2) & 1.72 & $0.66-4.52$ & 0.2669 & & & \\
\hline Disease location (not pancreas) & 8.54 & $2.90-25.15$ & $<0.0001$ & 9.64 & $2.71-34.35$ & 0.0005 \\
\hline Tumor type (benign) & 1.27 & $0.38-4.25$ & 0.6982 & & & \\
\hline Performed operation (PPPD) & 1.09 & $0.36-3.24$ & 0.8834 & & & \\
\hline Portal vein resection (yes) & 0.55 & $0.14-2.15$ & 0.3863 & & & \\
\hline Operation time ( $\geq 607 \mathrm{~min})$ & 0.77 & $0.30-1.96$ & 0.5844 & & & \\
\hline Blood loss ( $\geq 1520 \mathrm{ml})$ & 1.52 & $0.59-3.86$ & 0.3841 & & & \\
\hline \multicolumn{7}{|l|}{ Preoperative plasma ghrelin $(\mathrm{fmol} / \mathrm{ml})$} \\
\hline $\mathrm{AG}(<10.6)$ & 1.79 & $0.58-5.52$ & 0.3142 & & & \\
\hline DG $(<41.6)$ & 3.09 & $1.16-8.28$ & 0.0246 & 2.21 & $0.61-8.08$ & 0.2289 \\
\hline Total ghrelin $(<47.3)$ & 2.60 & $0.99-6.81$ & 0.0523 & & & \\
\hline DG-to-AG ratio $(<3.1)$ & 6.23 & $1.98-19.67$ & 0.0018 & 5.34 & $1.19-24.03$ & 0.0292 \\
\hline
\end{tabular}

HR, hazard ratio; CI, confidence interval; BMI, body mass index; PNI, prognostic nutritional index; HS-mGPS, high-sensitivity modified Glasgow Prognostic Score; PPPD, pylorus-preserving pancreaticoduodenectomy; AG, acyl ghrelin; DG, desacyl ghrelin.
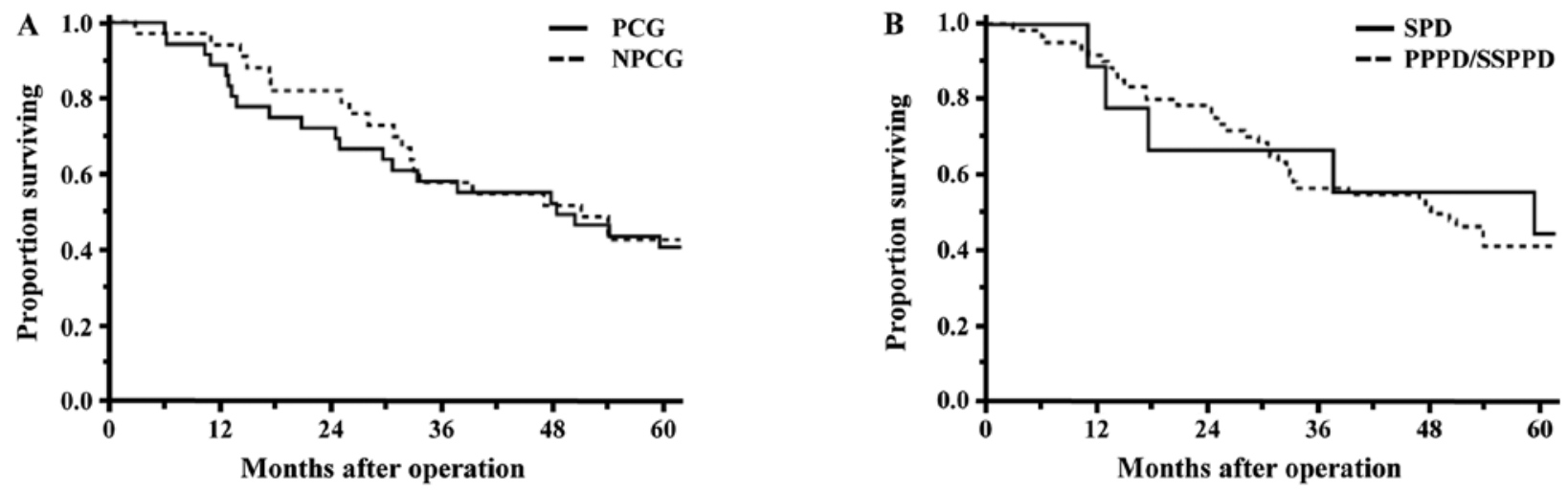

Figure 3. Survival curves for the 69 patients who underwent pancreaticoduodenectomy for malignant tumors. (A) Survival rates between the PCG and NPCG groups were not significantly different (5-year overall survival, 40.7\% vs. 42.4\%; P=0.8773). (B) Survival rates between SPD and PPPD/SSPPD were also not significantly different (5-year overall survival, 44.4\% vs. 41.0\%; $\mathrm{P}=0.8199$ ). PCG, pancreatic carcinoma group; NPCG, non-pancreatic carcinoma group; SPD, standard pancreatoduodenectomy; PPPD, pylorus-preserving pancreatoduodenectomy; SSPPD, subtotal stomach-preserving pancreatoduodenectomy.

study, H. pylori infection and the brevity of the period from gastrectomy to PD may have affected the delayed recovery of plasma ghrelin. Suppression of plasma ghrelin has been reported in patients with $H$. pylori (28). Takachi et al (10) indicated the possibility of compensatory ghrelin production in the remnant stomach and duodenum. As ghrelin does not recover in patients with total gastrectomy $(10,27)$, the influence of the residual stomach is significant, and a significant decline in ghrelin after SPD also suggests an association with duodenal compensation. The decrease in D/A 6 months after surgery may be the effect of decompensation due to residual stomach and duodenum, and recovery 1 year after surgery may be related to the restoration of compensation by other organs.

The PNI and HS-mGPS used in the present study are known as prognostic factors for patients with cancer (15-18). Plasma ghrelin and PNI in this study showed an inverse association. In the HS-mGPS, ghrelin is inversely associated with serum albumin, a nutritional indicator, irrespective of the inflammatory state. Ghrelin promotes food intake, and an inverse association between plasma ghrelin concentration and 
Table IV. Univariate and multivariate analyses of prognostic factors $(n=69)$.

\begin{tabular}{|c|c|c|c|c|}
\hline \multirow[b]{2}{*}{ Variables } & \multicolumn{2}{|c|}{ Univariate } & \multicolumn{2}{|c|}{ Multivariate } \\
\hline & P-value & HR & $95 \% \mathrm{CI}$ & P-value \\
\hline Age ( $\geq 69$ years) & 0.1924 & & & \\
\hline Sex (male) & 0.5077 & & & \\
\hline $\mathrm{BMI}\left(\geq 21.2 \mathrm{~kg} / \mathrm{m}^{2}\right)$ & 0.0349 & 1.23 & $0.62-2.48$ & 0.5568 \\
\hline Diabetes mellitus (yes) & 0.0002 & 2.79 & $1.37-5.75$ & 0.0046 \\
\hline Preoperative biliary drainage (yes) & 0.3491 & & & \\
\hline PNI $(<40.0)$ & 0.4849 & & & \\
\hline HS-mGPS (1+2) & 0.5406 & & & \\
\hline \multicolumn{5}{|l|}{ Tumor marker } \\
\hline $\mathrm{CEA}(\geq 4.9 \mathrm{ng} / \mathrm{ml})$ & 0.0464 & 2.07 & $0.89-4.56$ & 0.0898 \\
\hline CA19-9 $(\geq 500.0 \mathrm{U} / \mathrm{ml})$ & 0.0094 & 1.42 & $0.66-2.90$ & 0.3589 \\
\hline Tumor location (not pancreas) & 0.8773 & & & \\
\hline Tumor size $(\geq 30 \mathrm{~mm})$ & 0.0101 & 2.20 & $1.02-4.75$ & 0.0452 \\
\hline Pathological depth of invasion (T4) & 0.0095 & 1.35 & $0.67-2.67$ & 0.3987 \\
\hline Lymph node metastasis (yes) & 0.1049 & & & \\
\hline Stage (III) & 0.0816 & & & \\
\hline Performed operation (PPPD) & 0.1921 & & & \\
\hline Portal vein resection (yes) & 0.2831 & & & \\
\hline Operation time ( $\geq 631 \mathrm{~min})$ & 0.0478 & 1.01 & $0.47-2.23$ & 0.9771 \\
\hline Blood loss $(\geq 1580 \mathrm{ml})$ & 0.0224 & 1.34 & $0.57-3.10$ & 0.5006 \\
\hline CD score $(\geq \mathrm{III})$ & 0.3294 & & & \\
\hline Postoperative hospital stay ( $\geq 36$ days) & 0.0336 & 2.09 & $1.13-3.96$ & 0.0181 \\
\hline Adjuvant chemotherapy (yes) & 0.5544 & & & \\
\hline \multicolumn{5}{|l|}{ Preoperative plasma ghrelin (fmol/ml) } \\
\hline $\mathrm{AG}(<10.6)$ & 0.1529 & & & \\
\hline DG $(<41.6)$ & 0.0950 & & & \\
\hline Total ghrelin $(<47.3)$ & 0.2743 & & & \\
\hline DG-to-AG ratio $(<3.1)$ & 0.0134 & 3.06 & $1.34-6.76$ & 0.0089 \\
\hline
\end{tabular}

BMI has been shown $(29,30)$. Our results can be explained that fasting due to preoperative jaundice and cholangitis develops into malnutrition, and as a result, ghrelin increased as positive feedback in the low PNI group and HS-mGPS score 2 group. In the present study, PNI and HS-GPS were not independent factors in the analyses of risk factors of morbidity and mortality. Depending on the control of obstructive jaundice and cholangitis, the high inflammatory state can last and progress to malnutrition and weight loss (31). It is speculated that the relatively good control of jaundice and cholangitis in the present study patients affected the analyses between the PNI and HS-GPS and outcomes. The D/A tended to be higher in the good group of conditions, whether PNI or HS-mGPS, as opposed to the inversely associated plasma ghrelin concentrations. It may be that DG which is still insufficiently researched is involved.

As mentioned above, plasma ghrelin, especially AG and $\mathrm{TG}$, has been reported to be associated with surgery outcomes.
The present study focused specifically on D/A because it included SPD patients with significantly lower plasma ghrelin levels. There was no significant difference between morbidity and mortality between the SPD group and Non-SPD group. Therefore, we suggest that relative evaluation by D/A is important rather than evaluation based on the absolute value of plasma ghrelin. In the multivariate analyses of morbidity and mortality, a lower D/A was an independent risk factor. A high preoperative AG level is the result of feedback on malnutrition and inflammatory response and may cause postoperative complications and a poor prognosis. Previous studies reported that ghrelin associated with cancer cell proliferation, invasion and migration in cell lines of pancreatic, breast, renal and colorectal cancer (32-35). The correlation of the ghrelin gene and GHSR expression in cancer patients with prognosis was also reviewed (36). However, AG alone cannot be a risk factor, and the involvement of DG is indispensable. Some reports indicated that DG protects cardiomyocytes from ischemic 

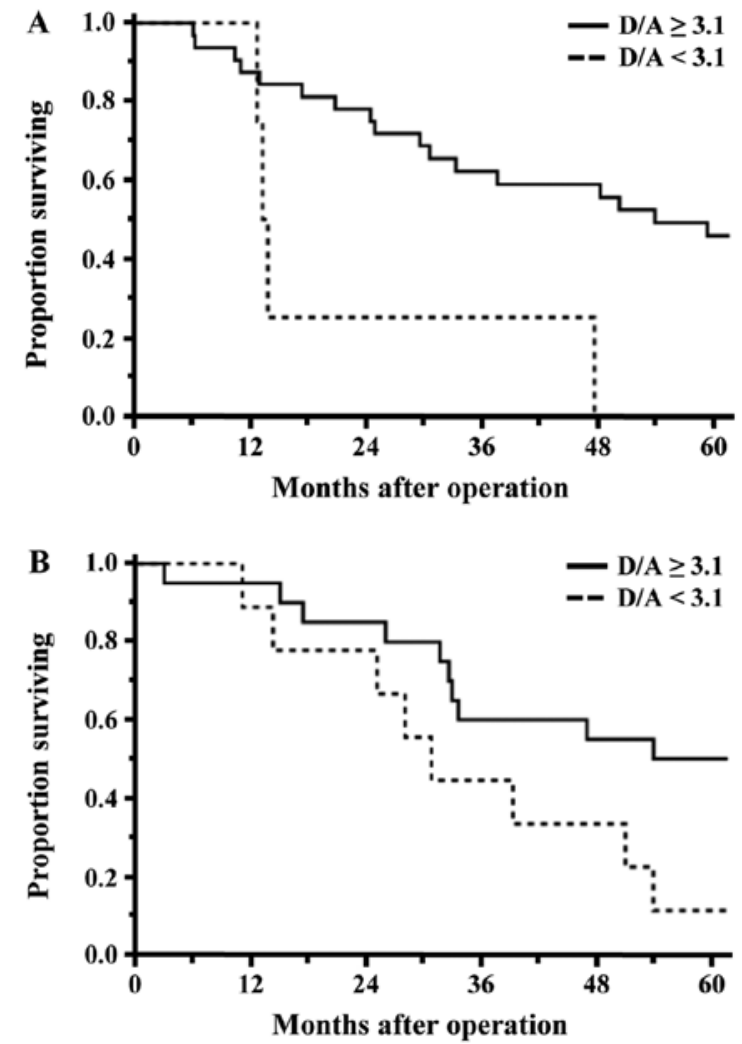

Figure 4. Comparison of overall survival curves between the low and high D/A groups of patients with malignant tumors. (A) The 5-year survival of patients with pancreatic carcinoma differed significantly between the two groups (low $\mathrm{D} / \mathrm{A} 0.0 \%$ vs. high $\mathrm{D} / \mathrm{A} 45.8 \% ; \mathrm{P}=0.0096$ ). (B) The 5 -year survival in non-pancreatic carcinomas differed significantly between the two groups (low D/A $11.1 \%$ vs. high D/A 50.0\%; $\mathrm{P}=0.0247$ ). D/A, desacyl ghrelin-to-acyl ghrelin ratio.

injury and promotes muscle regeneration and inhibit apoptosis in myoblasts cocultured with colon carcinoma cells $(37,38)$. Specific receptors of DG that promote these activities have not been identified, and a feedback mechanism such as that with $\mathrm{AG}$ is unknown. Further research on the relationship with AG is also necessary.

One limitation of the present study is that the background of the subjects is unbalanced. The disease indication for PD is not unified, the tumor factors are irregular, and only a very few patients underwent SPD or SSPPD with resection of the stomach. Although not presented in this report, the tumor size, diabetes mellitus and postoperative hospital stay listed as poor prognosis factors other than D/A ratio were particularly involved in the PCG. Another limitation is the insufficient extraction of factors that affect ghrelin production and feedback. In addition to nutrition and inflammation, $H$. pylori infection and cachexia status affect ghrelin production $(28,39,40)$. These factors cannot be ignored because the rate of $H$. pylori infection is higher in Japanese people, and the progression of pancreatic and biliary carcinoma is strongly related to cachexia $(41,42)$.

This study showed the D/A to be a potentially useful predictive factor for postoperative complications and prognosis after PD. Based on the outcomes of SPD cases with decreased preoperative plasma ghrelin, the absolute value of plasma ghrelin alone cannot explain complications or prognosis.
Because of the limitations of a small sample size and the single-institution nature of this study, it will be necessary to conduct a prospective, large, multicenter trial in patients to fully take into consideration patient, tumor and surgical factors. The mechanism of the physiological activity of DG must be elucidated in the next step.

\section{Acknowledgements}

The authors would like to thank Professor Kazuo Chijiiwa (Department of Surgery, University of Miyazaki Faculty of Medicine, deceased November 26, 2013) for designing the present study and performing surgery. The authors would like to acknowledge Dr Jiro Ohuchida (Department of Surgery, Miyazaki Prefectural Miyazaki Hospital), Dr Motoaki Nagano (Department of Surgery, Miyazaki Medical Center Hospital) and Dr Yoshiro Fujii (Department of Surgery, Yokohama City University Medical Center) who assisted with performed the surgery, blood sampling and follow-up care.

\section{Funding}

No funding was received.

\section{Availability of data and materials}

The datasets used and analyzed during the present study are available from the corresponding author on reasonable request.

\section{Authors' contributions}

TN performed the surgery, analyzed the data and wrote the manuscript. HT measured plasma ghrelin levels and analyzed the data. TH performed the surgery and analyzed the data. NI, MH and KY performed the surgery, blood sampling and follow-up care. AN contributed to surgical and survival analyses, univariate/multivariate analyses and reviewed the manuscript. KK and MN conceived and designed the current study, measured plasma ghrelin levels, analyzed plasma ghrelin and immunonutritional status and critically revised the manuscript. All authors read and approved the final version of the manuscript.

\section{Ethics approval and consent to participate}

The present study was approved by the Human Ethics Review Board of the University of Miyazaki (approval no. O-0426) and conformed to the provisions of the Declaration of Helsinki. Informed consent for data collection was obtained from all patients using an opt-out procedure. Patient data were retrieved from the departmental database.

\section{Patient consent for publication}

Patient consent for publication was obtained from the patients using the opt-out procedure.

\section{Competing interests}

The authors declare that they have no competing interests. 


\section{References}

1. Diener MK, Knaebel HP, Heukaufer C, Antes G, Büchler MW and Seiler CM: A systematic review and meta-analysis of pylorus-preserving versus classical pancreaticoduodenectomy for surgical treatment of periampullary and pancreatic carcinoma. Ann Surg 245: 187-200, 2007.

2. Huang W, Xiong JJ, Wan MH, Szatmary P, Bharucha S, Gomatos I, Nunes QM, Xia Q, Sutton R and Liu XB: Meta-analysis of subtotal stomach-preserving pancreaticoduodenectomy vs. pylorus preserving pancreaticoduodenectomy. World J Gastroenterol 21: 6361-6373, 2015

3. Watson K: Carcinoma of ampulla of vater successful radical resection. Br J Surg 31: 368-373, 1944.

4. Traverso LW and Longmire WJ: Preservation of the pylorus in pancreaticoduodenectomy. Surg Gynecol Obstet 146: 959-962, 1978.

5. Hiyoshi M, Chijiiwa K, Ohuchida J, Imamura $\mathrm{N}$ and Nagano M: Comparative study of gastric emptying and nutritional status after pylorus-preserving vs. subtotal stomach-preserving pancreaticoduodenectomy. Hepatogastroenterology 59: 1018-1022, 2012.

6. Zhao R, Chang Y, Wang X, Zhang $\mathrm{P}$, Zhang $\mathrm{C}$ and Lian $\mathrm{P}$ Pylorus-preserving pancreaticoduodenectomy versus standard pancreaticoduodenectomy in the treatment of duodenal papilla carcinoma. Oncol Lett 15: 6368-6376, 2018.

7. Kojima M, Hosoda H, Date Y, Nakazato M, Matsuo H and Kangawa K: Ghrelin is a growth-hormone-releasing acylated peptide from stomach. Nature 402: 656-660, 1999.

8. Asakawa A, Inui A, Kaga T, Yuzuriha H, Nagata T, Ueno N, Makino S, Fujimiya M, Niijima A, Fujino MA and Kasuga M: Ghrelin is an appetite-stimulatory signal from stomach with structural resemblance to motilin. Gastroenterology 120: 337-345, 2001.

9. Muccioli G, Tschöp M, Papotti M, Deghenghi R, Heiman M and Ghigo E: Neuroendocrine and peripheral activities of ghrelin: Implications in metabolism and obesity. Eur J Pharmacol 440: 235-254, 2002.

10. Takachi K, Doki Y, Ishikawa O, Miyashiro I, Sasaki Y, Ohigashi H, Murata K, Nakajima H, Hosoda H, Kangawa K, et al: Postoperative ghrelin levels and delayed recovery from body weight loss after distal or total gastrectomy. J Surg Res 130: 1-7, 2006

11. Doki Y,Takachi K, Ishikawa O, Miyashiro I, Sasaki Y, OhigashiH, Nakajima H, Hosoda H, Kangawa K, Sasakuma F, et al: Ghrelin reduction after esophageal substitution and its correlation to postoperative body weight loss in esophageal cancer patients. Surgery 139: 797-805, 2006.

12. Takiguchi S, Murakami K, Yanagimoto Y, Takata A, Miyazaki Y, Mori M and Doki Y: Clinical application of ghrelin in the field of surgery. Surg Today 45: 801-807, 2015.

13. Tsuchimochi W, Kyoraku I, Yamaguchi H, Toshinai K, Shiomi K, Kangawa K and Nakazato M: Ghrelin prevents the development of experimental diabetic neuropathy in rodents. Eur J Pharmacol 702: 187-193, 2013

14. Yano Y, Nakazato M, Toshinai K, Inokuchi T, Matsuda S, Hidaka T, Hayakawa M, Kangawa K, Shimada K and Kario K Circulating des-acyl ghrelin improves cardiovascular risk prediction in older hypertensive patients. Am J Hypertens 27: 727-733, 2014.

15. Kanda M, Fujii T, Kodera Y, Nagai S, Takeda S and Nakao A: Nutritional predictors of postoperative outcome in pancreatic cancer. Br J Surg 98: 268-274, 2011.

16. Zhang P, Xi M, Li QQ, He LR, Liu SL, Zhao L, Shen JX and Liu MZ: The modified glasgow prognostic score is an independent prognostic factor in patients with inoperable thoracic esophageal squamous cell carcinoma undergoing chemoradiotherapy. J Cancer 5: 689-695, 2014.

17. Chen P, Fang M, Wan Q, Zhang X, Song $T$ and Wu $S$ : High-sensitivity modified Glasgow prognostic score (HS-mGPS) is superior to the mGPS in esophageal cancer patients treated with chemoradiotherapy. Oncotarget 8: 99861-99870, 2017.

18. Cui Y, Li J, Cao YH, Liu MY, Shi ZX and Gao TH: Predictive and prognostic significance of high-sensitivity modified glasgow prognostic score (HS-mGPS) in advanced gastric cancer patients treated with neoadjuvant chemotherapy. Zhonghua Zhong Liu Za Zhi 39: 195-200, 2017 (In Chinese).

19. Brierley JD, Gospodarowicz MK and Wittekind C (eds): TNM classification of malignant tumors, 8th edition. Wiley-Blackwell, 2017.
20. Dindo D, Demartines N and Clavien PA: Classification of surgical complications: A new proposal with evaluation in a cohort of 6336 patients and results of a survey. Ann Surg 240: 205-213, 2004

21. Hiyoshi M, Chijiiwa K, Fujii Y, Imamura N, Nagano M and Ohuchida J: Usefulness of drain amylase, serum C-reactive protein levels and body temperature to predict postoperative pancreatic fistula after pancreaticoduodenectomy. World J Surg 37: 2436-2442, 2013

22. Kakita A, Yoshida M and Takahashi T: History of pancreaticojejunostomy in pancreaticoduodenectomy: Development of a more reliable anastomosis technique. J Hepatobiliary Pancreat Surg 8: 230-237, 2001.

23. Chijiiwa K, Imamura N, Ohuchida J, Hiyoshi M, Nagano M, Otani K, Kai M and Kondo K: Prospective randomized controlled study of gastric emptying assessed by (13)C-acetate breath test after pylorus-preserving pancreaticoduodenectomy: Comparison between antecolic and vertical retrocolic duodenojejunostomy. J Hepatobiliary Pancreat Surg 16: 49-55, 2009.

24. Uesaka K, Boku N, Fukutomi A, Okamura Y, Konishi M, Matsumoto I, Kaneoka Y, Shimizu Y, Nakamori S, Sakamoto $\mathrm{H}$, et al: Adjuvant chemotherapy of S-1 versus gemcitabine for resected pancreatic cancer: A phase 3, open-label, randomised, non-inferiority trial (JASPAC 01). Lancet 388 : 248-257, 2016

25. Nanashima A, Murakami G, Takagi K, Arai J, Sumida Y, Kodama $\mathrm{T}$ and Nagayasu T: Relationship between serum ghrelin level and physiology in patients who underwent hepatectomy and pancreatectomy. Acta Med Nagasaki 61: 55-60, 2017.

26. Sasaki K, Asaoka T, Eguchi H, Fukuda Y, Iwagami Y, Yamada D, Miyazaki Y, Noda T, Takahashi T, Gotoh K, et al: Plasma ghrelin suppression as an early predictor for postoperative complications after pancreatoduodenectomy. Pancreatology 18 : 73-78, 2018

27. Takiguchi S, Takata A, Murakami K, Miyazaki Y, Yanagimoto Y, Kurokawa Y, Takahashi T, Mori M and Doki Y: Clinical application of ghrelin administration for gastric cancer patients undergoing gastrectomy. Gastric Cancer 17: 200-205, 2014.

28. Kasai C, Sugimoto K, Moritani I, Tanaka J, Oya Y, Inoue H, Tameda M, Shiraki K, Ito M, Takei Y and Takase K: Changes in plasma ghrelin and leptin levels in patients with peptic ulcer and gastritis following eradication of Helicobacter pylori infection. BMC Gastroenterol 16: 119, 2016.

29. Shiiya T, Nakazato M, Mizuta M, Date Y, Mondal MS, Tanaka M, Nozoe S, Hosoda H, Kangawa K and Matsukura S: Plasma ghrelin levels in lean and obese humans and the effect of glucose on ghrelin secretion. J Clin Endocrinol Metab 87: 240-244, 2002

30. Date Y, Murakami N, Toshinai K, Matsukura S, Niijima A, Matsuo H, Kangawa K and Nakazato M: The role of the gastric afferent vagal nerve in ghrelin-induced feeding and growth hormone secretion in rats. Gastroenterology 123: 1120-1128, 2002.

31. Kumamoto Y, Kaizu T, Tajima H, Nishizawa N, Ei S, Igarashi K and Watanabe M: Neutrophil-to-lymphocyte ratio as a predictor of postoperative morbidity in patients with distal cholangiocarcinoma. Mol Clin Oncol 9: 362-368, 2018.

32. Duxbury MS, Waseem T, Ito H, Robinson MK, Zinner MJ, Ashley SW and Whang EE: Ghrelin promotes pancreatic adenocarcinoma cellular proliferation and invasiveness. Biochem Biophys Res Commun 309: 464-468, 2003.

33. Jeffery PL, Murray RE, Yeh AH, McNamara JF, Duncan RP, Francis GD, Herington AC and Chopin LK: Expression and function of the ghrelin axis, including a novel preproghrelin isoform, in human breast cancer tissues and cell lines. Endocr Relat Cancer 12: 839-850, 2005.

34. Lin TC, Liu YP, Chan YC, Su CY, Lin YF, Hsu SL, Yang CS and Hsiao M: Ghrelin promotes renal cell carcinoma metastasis via Snail activation and is associated with poor prognosis J Pathol 237: 50-61, 2015.

35. Waseem T, Javaid-Ur-Rehman, Ahmad F, Azam M and Qureshi MA: Role of ghrelin axis in colorectal cancer: A novel association. Peptides 29: 1369-1376, 2008.

36. Lin TC and Hsiao M: Ghrelin and cancer progression. Biochim Biophys Acta Rev Cancer 1868: 51-57, 2017.

37. Yanagi S, Sato T, Kangawa $\mathrm{K}$ and Nakazato $\mathrm{M}$ : The homeostatic force of ghrelin. Cell Metab 27: 786-804, 2018.

38. Zeng X, Chen S, Lin Y and Ke Z: Acylated and unacylated ghrelin inhibit apoptosis in myoblasts cocultured with colon carcinoma cells. Oncol Rep 39: 1387-1395, 2018 
39. Garcia JM, Garcia-Touza M, Hijazi RA, Taffet G, Epner D, Mann D, Smith RG, Cunningham GR and Marcelli M: Active ghrelin levels and active to total ghrelin ratio in cancer-induced cachexia. J Clin Endocrinol Metab 90: 2920-2926, 2005.

40. Kerem M, Ferahkose Z, Yilmaz UT, Pasaoglu H, Ofluoglu E, Bedirli A, Salman B, Sahin TT and Akin M: Adipokines and ghrelin in gastric cancer cachexia. World J Gastroenterol 14: 3633-3641, 2008.
41. Ozola Zalite I, Zykus R, Francisco Gonzalez M, Saygili F, Pukitis A, Gaujoux S, Charnley RM and Lyadov V: Influence of cachexia and sarcopenia on survival in pancreatic ductal adenocarcinoma: A systematic review. Pancreatology 15: 19-24, 2015.

42. Abe K, Uwagawa T, Haruki K, Takano Y, Onda S, Sakamoto T, Gocho $T$ and Yanaga K: Effects of $\omega-3$ fatty acid supplementation in patients with bile duct or pancreatic cancer undergoing chemotherapy. Anticancer Res 38: 2369-2375, 2018. 